\title{
Phosphatase PP2A enhances MCL-1 protein half-life in multiple myeloma cells
}

\author{
Anne Slomp ${ }^{1}$, Laura M. Moesbergen ${ }^{1}$, Eric Eldering ${ }^{2,3}$, Marie José Kersten ${ }^{3,4}$, Monique C. Minnema ${ }^{5}$ and \\ Victor Peperzak'
}

\begin{abstract}
Multiple myeloma (MM), a treatable but incurable malignancy, is characterized by the growth of clonal plasma cells in protective niches in the bone marrow. MM cells depend on expression of BCL-2 family proteins, in particular MCL-1, for survival. The regulation of MCL-1 is complex and cell type-dependent. Unraveling the exact mechanism by which MCL-1 is overexpressed in MM may provide new therapeutic strategies for inhibition in malignant cells, preferably limiting side effects in healthy cells. In this study, we reveal that one cause of overexpression could be stabilization of the MCL-1 protein. We demonstrate this in a subset of $\mathrm{MM}$ and diffuse large $B$ cell lymphoma (DLBCL) cell lines and MM patient samples. We applied a phosphatase siRNA screen to identify phosphatases responsible for MCL-1

stabilization in MM, and revealed PP2A as the MCL-1 stabilizing phosphatase. Using the PP2A inhibitor okadaic acid, we validated that PP2A dephosphorylates MCL-1 at Ser159 and/or Thr163, and thereby stabilizes MCL-1 in MM cells with long MCL-1 half-life, but not in DLBCL cells. Combined kinase and phosphatase inhibition experiments suggest that the MCL-1 half-life in MM is regulated by the counteracting functions of JNK and PP2A. These findings increase the understanding of the mechanisms by which MCL-1 is post-translationally regulated, which may provide novel strategies to inhibit MCL-1 in MM cells.
\end{abstract}

\section{Introduction}

Pro-survival B cell lymphoma 2 (BCL-2) family proteins are potent inhibitors of programmed cell death and are often overexpressed in malignant cells, enabling these cells to survive in the presence of DNA damage, abnormal growth signaling, and chemotherapeutic agents ${ }^{1}$. MCL-1 is overexpressed in many germinal center-derived malignancies, including multiple myeloma (MM), diffuse large B cell lymphoma (DLBCL), and follicular lymphoma ${ }^{2}$. MCL-1 expression is essential for survival of $\mathrm{MM}^{3-5}$, and its overexpression in $\mathrm{MM}$ is associated with relapse and shorter survival ${ }^{6}$, making MCL-1 an attractive therapeutic target. Multiple MCL-1-specific BH3-mimetics have

\footnotetext{
Correspondence: Victor Peperzak (v.peperzak@umcutrecht.nl)

${ }^{1}$ Center for Translational Immunology, University Medical Center Utrecht, Utrecht University, Utrecht, The Netherlands

${ }^{2}$ Department of Experimental Immunology, Amsterdam University Medical

Centers, University of Amsterdam, Amsterdam, The Netherlands

Full list of author information is available at the end of the article

Edited by M. Herold
}

shown effectivity in preclinical models and are now being evaluated in clinical trials for MM and other hematologic malignancies $^{2,7-9}$. Thus, targeting MCL-1 may be beneficial for the treatment of B cell malignancies such as MM. However, in addition to its importance for malignant cells, MCL-1 expression is important for the survival of many healthy cells and tissues, such as plasma cells ${ }^{10}, \mathrm{~B}$ cells $^{11,12}$, hematopoietic stem cells ${ }^{13}$, cardiomyocytes ${ }^{14}$, and neural precursor cells ${ }^{15}$. In order to prevent unwanted side effects of MCL-1 inhibition on healthy tissues, it is important to unravel the exact mechanism of MCL-1 upregulation in $\mathrm{MM}$, because this may provide new strategies to inhibit MCL-1 in malignant cells.

MCL-1 distinguishes itself from other BCL-2 family members by its size and structure. Although the Cterminal part of MCL-1, which contains a BH3 domain that is required for its anti-apoptotic function, closely resembles other pro-survival BCL-2 family members, MCL-1 is considerably larger and has an N-terminus that

\section{(c) The Author(s) 2021}

(c) (i) Open Access This article is licensed under a Creative Commons Attribution 4.0 International License, which permits use, sharing, adaptation, distribution and reproduction cc) in any medium or format, as long as you give appropriate credit to the original author(s) and the source, provide a link to the Creative Commons license, and indicate if changes were made. The images or other third party material in this article are included in the article's Creative Commons license, unless indicated otherwise in a credit line to the material. If material is not included in the article's Creative Commons license and your intended use is not permitted by statutory regulation or exceeds the permitted use, you will need to obtain permission directly from the copyright holder. To view a copy of this license, visit http://creativecommons.org/licenses/by/4.0/. 
is subject to extensive post-translational modification. The presence of PEST regions (enriched in proline (P), glutamic acid $(\mathrm{E})$, serine $(\mathrm{S})$, and threonine $(\mathrm{T})$ residues), as well as the presence of four arginine pairs, allows for rapid turnover of MCL-1 (refs. ${ }^{16,17}$ ), resulting in a halflife of $30-40 \mathrm{~min}$ in many cell types ${ }^{18,19}$. Proteasomal degradation of MCL-1 occurs upon phosphorylation ${ }^{20}$ and subsequent poly-ubiquitination ${ }^{21}$ of its PEST regions.

In its PEST regions, MCL-1 contains at least 13 putative phosphorylation sites, some of which have been experimentally studied and verified ${ }^{20}$. Kinases GSK-3 $\beta$, ERK-1, and JNK have been shown to phosphorylate MCL-1 at Thr163, and subsequently Ser155 and Ser159, resulting in MCL-1 destabilization ${ }^{20,22-25}$. Multiple ubiquitin ligases have been associated with MCL-1 destabilization, including Mule, $\mathrm{SCF}^{\beta-\mathrm{TrCP}}, \mathrm{SCF}^{\mathrm{Fbw} 7}, \mathrm{APC} / \mathrm{C}^{\mathrm{Cdc} 20}$, and Trim17 (refs. ${ }^{21,25-29}$ ). The deubiquitinases USP9X ${ }^{30}$, Ku70 (ref. ${ }^{31}$ ), and USP13 (ref. ${ }^{32}$ ) were shown to counteract this process and thereby stabilize MCL-1. Modification of MCL-1 by kinases and ubiquitin modifiers is highly dependent on cellular context, and the exact role and mechanism of MCL-1 stabilization in MM are unknown.

In healthy germinal center (GC) B cells, tyrosine and serine/threonine phosphatase activity was shown to be increased compared to non-GC and naïve $B$ cells ${ }^{33}$. It was also observed that stability of MCL-1 is increased in GC B cells, resulting in increased protein levels, and that this increased MCL-1 stability could be reversed by treatment with okadaic acid (OA), an inhibitor targeting the PP2A phosphatase complex ${ }^{34}$. We hypothesize that MCL-1 overexpression in post-GC B cell malignancies is the result of increased dephosphorylation of MCL-1, which leads to stabilization of the protein. Identifying the specific phosphatase responsible for MCL-1 dephosphorylation would increase insight into MCL-1 protein regulation and may result in the identification of new treatment targets for B cell malignancies.

In this study, we show that MCL-1 is stabilized in a subset of DLBCL and MM cell lines and primary MM cells. By using a phosphatase siRNA screen, we identify PP2A as MCL-1 stabilizing phosphatase in MM, but not in DLBCL cells. Moreover, we show that in MM, PP2A activity reverses phosphorylation of MCL-1 by JNK.

\section{Materials and methods \\ Cell culture}

Cell lines L363, OPM-2, MM1.S, RPMI-8226, and SUDHL-2 were cultured in RPMI 1640 GlutaMAX HEPES culture medium (Life Technologies) supplemented with $10 \%$ fetal bovine serum (FBS, Biowest) and $100 \mu \mathrm{g} / \mathrm{ml}$ penicillin-streptomycin (Gibco/Life Technologies). UM9 was cultured with $20 \% \mathrm{FBS}$, and NCI-H929 with $20 \% \mathrm{FBS}$, $1 \mathrm{mM}$ sodium pyruvate (Thermo Fisher), and $50 \mu \mathrm{M}$ $\beta$-mercaptoethanol (Life Technologies). OCI-Ly1, OCI-Ly3, OCI-Ly7, and OCI-Ly10 were cultured in Iscove's modified Dulbecco's medium (Life Technologies) supplemented with $20 \% \mathrm{FBS}$ and $100 \mu \mathrm{g} / \mathrm{ml}$ penicillin-streptomycin. Cell lines L363, RPMI-8226, OPM-2, OCI-Ly3, OCI-Ly7, OCI-Ly1 and NCI-H929 were purchased from DSMZ, MM1.S, OCILy10, and SU-DHL-2 were purchased from ATCC. Cell line UM9 was derived from an MM patient at our UMCU hospital). All cell lines were tested negative for mycoplasma before and during the experiments. Primary MM cell samples were derived from patients diagnosed at the Academic Medical Center, Amsterdam, The Netherlands. This study was conducted and approved by the AMC Medical Committee on Human Experimentation. Informed consent was obtained in accordance with the Declaration of Helsinki.

\section{Chemicals}

In order to assess the effect of PP1 and PP2A phosphatases, cells were treated for $2 \mathrm{~h}$ (unless otherwise stated) with $1 \mu \mathrm{M}$ tautomycetin (TMC) (2305, Tocris) or 100 nM OA (ALX-350-003; Enzo Life Sciences), respectively. When phosphorylation of MCL-1 was studied, all samples were treated with $20 \mathrm{nM}$ bortezomib (S1013; Selleckchem) and $10 \mu \mathrm{M}$ Q-VD-OPh (SML0063; SigmaAldrich). Protein half-life determination was performed using $25 \mu \mathrm{g} / \mathrm{ml}$ cycloheximide (CHX) (HY-12320; MedChemExpress) for $0,1,2$, and $4 \mathrm{~h}$ or for $0.5,1$, and $2 \mathrm{~h}$. In case of CHX experiments with OA, cells were pretreated for $2 \mathrm{~h}$ with OA, and OA remained present during $\mathrm{CHX}$ treatment. In order to determine MCL-1 half-life in primary MM samples, CD138+ cells were purified from MM bone marrow aspirates by magnetic activated cell sorting using CD138 microbeads (Miltenyi Biotec), followed by CHX treatment as described above. CDK7 inhibitor THZ1 (MedChemExpress) was used at $200 \mathrm{nM}$ for $8 \mathrm{~h}$ in order to inhibit MCL1 transcription. Ten micromolar of GSK-3 $\beta$ inhibitor CHIR99021 (Tocris Bioscience), $250 \mathrm{nM}$ of MEK/ ERK-1 inhibitor trametinib/GSK1120212 (Selleckchem), and $20 \mu \mathrm{M}$ of JNK inhibitor SP600125 (Merck) were used to assess the role of kinases in MCL-1 degradation.

\section{Immunoblotting}

For western blot analysis, cells were lysed in buffer containing 1\% NP-40 and proteins were separated using SDS-PAGE (Mini-PROTEAN® TGX ${ }^{\mathrm{TM}}$ Precast Gels, BioRad), transferred to low fluorescence PVDF membranes (Bio-Rad), blocked in phosphate-buffered saline (PBS) containing $2 \%$ non-fat dry milk, and stained using the following antibodies: mouse anti- $\alpha$-tubulin, rabbit antiphospho-MCL-1 (Ser159/Thr163), rabbit anti-PP2A A subunit (PPP2R1A/B; Cell Signaling), mouse anti-PP2A C subunit, alpha isoform (PPP2CA; Merck), rabbit antiMCL-1 (Abcam), mouse anti-MCL-1 (Santa Cruz), goat 
anti-mouse-680RD, and goat anti-rabbit-800CW (LI-COR Biosciences). For phospho-MCL-1 immunoblotting, all PBS in washing and staining buffers was replaced with TBS. Infrared imaging was used for detection (Odyssey Sa; LI-COR Biosciences). Analysis and quantification were performed using LI-COR Image Studio and ImageJ 1.47V software.

\section{Quantitative PCR}

Total RNA for quantitative real-time PCR was extracted using RNA-Bee (Tel-Test, Inc.) according to the manufacturer's protocol. cDNA was produced using the RevertAid $\mathrm{H}$ minus first strand cDNA synthesis kit (K1632; Thermo Fisher Scientific), and qPCR was performed with SYBR Green PCR master mix (4309155; Life Technologies) using the ViiA 7 Real-Time PCR System (Thermo Fisher Scientific). The following primers were used: MCL1 forward 5'-TCGTAAGGACAAAACGGGA C-3', MCL1 reverse 5'-CATTCCTGATGCCACCTTCT3', HPRT forward 5'-CCTGGCGTCGTGATTAGTGA-3', HPRT reverse 5'-CGAGCAAGACGTTCAGTCCT-3', UBC forward 5'-ATTTGGGTCGCGGTTCTTG-3', UBC reverse $5^{\prime}$-TGCCTTGACATTCTCGATGGT-3', YWH AZ forward 5'-ACTTTTGGTACATTGTGGCTTCA A- $3^{\prime}$, and YWHAZ reverse 5'-CCGCCAGGACAAACC AGTAT-3'. For PPP2R2C predesigned Taqman probe sets where used (hs00902099_m1, Thermo Fisher Scientific). Results were normalized to expression of HPRT, UBC, and YWHAZ using the $\Delta \Delta C$ t method ${ }^{35}$.

\section{Gene expression data analysis}

Using published data sets, $P P P 2 R 2 C$ gene expression was assessed in different GC B cell-derived malignancies and in healthy GC B cells. The following data sets from the National Center for Biotechnology Information Gene Expression Omnibus were used: GSE2658, GSE93291, GSE93261, GSE39671, GSE87371, and GSE38697. Data sets were analyzed using the R2 Genomics Analysis and Visualization Platform.

\section{Phosphatase siRNA screen}

A library of siRNAs against 188 human phosphatases (Dharmacon ON-TARGETplus ${ }^{\circledR}$ SMARTpool ${ }^{\circledR}$ siRNA Library-Human Phosphatase) was transfected into MM1. $\mathrm{S}$ cells using Lipofectamine RNAiMAX and $50 \mathrm{nM}$ siRNA. Sixty-six hours after transfection, lysates were made for detection of MCL-1 protein levels by immunoblotting.

\section{Statistical analysis}

MCL-1 half-life was determined using a one-phase exponential decay non-linear regression analysis model (GraphPad Prism version 8.0.1). Statistical analysis was performed using GraphPad Prism version 8.0.1. Statistical differences between experimental groups were examined by a one-way analysis of variance (ANOVA) with Sidak post hoc multiple comparison testing for Fig. 5B and a two-way ANOVA with Holm-Sidak post hoc multiple comparison testing for Fig. S3. For all tests a $p$ value of $<0.05$ was considered statistically significant.

\section{Results}

MCL-1 is stabilized in post-GC B cell malignancies

The MCL-1 protein half-life is reported to be $30-40 \mathrm{~min}$ in many cell types and tissues ${ }^{18,19}$. By treating cell lines with CHX for different time periods, we determined the half-life of MCL-1 in a panel of MM and DLBCL cell lines (Fig. 1A-C). In some cell lines, such as MM cell lines L363, NCI-H929, OPM-2, and DLBCL cell lines OCI-Ly7, OCI-Ly1, and SU-DHL-2, the MCL-1 halflife is relatively short and in line with published half-lives. In contrast, MM cell lines MM1.S, RPMI-8226, and UM9, as well as DLBCL cell lines OCI-Ly10 and OCI-Ly3 are characterized by more stable MCL-1, with a half-life significantly longer than $40 \mathrm{~min}$. In order to determine whether stabilization of MCL-1 is also observed in primary MM cells, the half-life of MCL-1 was determined in CD138-purified cells from MM bone marrow aspirates (Fig. 1D). In five tested patient samples, the MCL-1 halflife ranged from 32 to $177 \mathrm{~min}$.

\section{MCL-1 protein level reflects transcriptional activity as well as protein half-life}

In order to investigate whether increased MCL-1 stability is mirrored by an increased amount of protein, we determined MCL-1 protein expression across the MM and DLBCL cell line panel (Fig. 2A). In the DLBCL cell lines, the amount of MCL-1 protein seems to correlate well with protein stability (Fig. 2B). This was not the case in the MM cell lines, where high MCL-1 protein levels were observed in NCI-H929, while this cell line has a normal MCL-1 half-life. If high MCL-1 protein levels in NCI-H929 are not caused by protein stabilization, they are likely to result from high $M C L 1$ transcription. To address this, the rate of $M C L 1$ transcription was determined by reverse transcriptase PCR (RT-PCR) analysis for each cell line, and both the rate of transcription and protein half-life of MCL-1 were compared to the total MCL1 protein level (Fig. 2B). This analysis reveals that high MCL-1 protein expression in MM cells can be a consequence of high gene transcription, increased protein half-life, or both. In contrast to MM, MCL-1 protein expression in DLBCL cells seems to be mainly correlated to protein half-life.

\section{Phosphatase siRNA screen identifies PP2A as MCL-1- stabilizing phosphatase complex}

Phosphatases stabilizing MCL-1 may be interesting therapeutic targets because their inhibition is expected to 
A

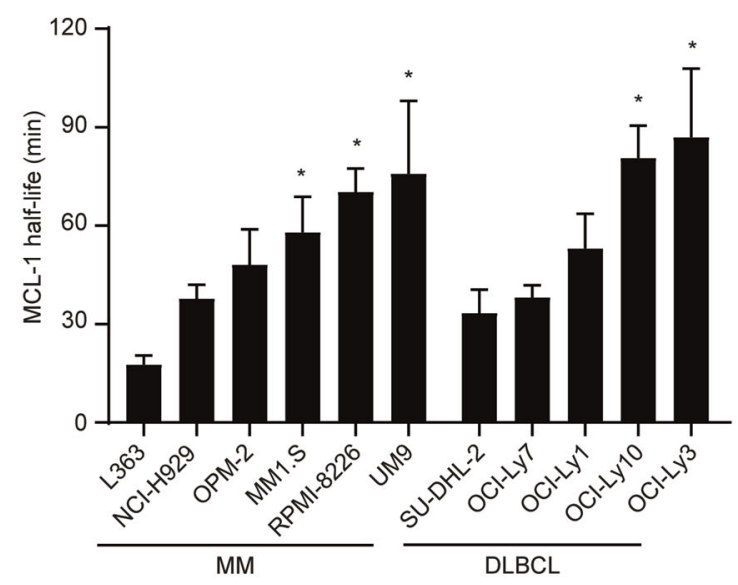

B
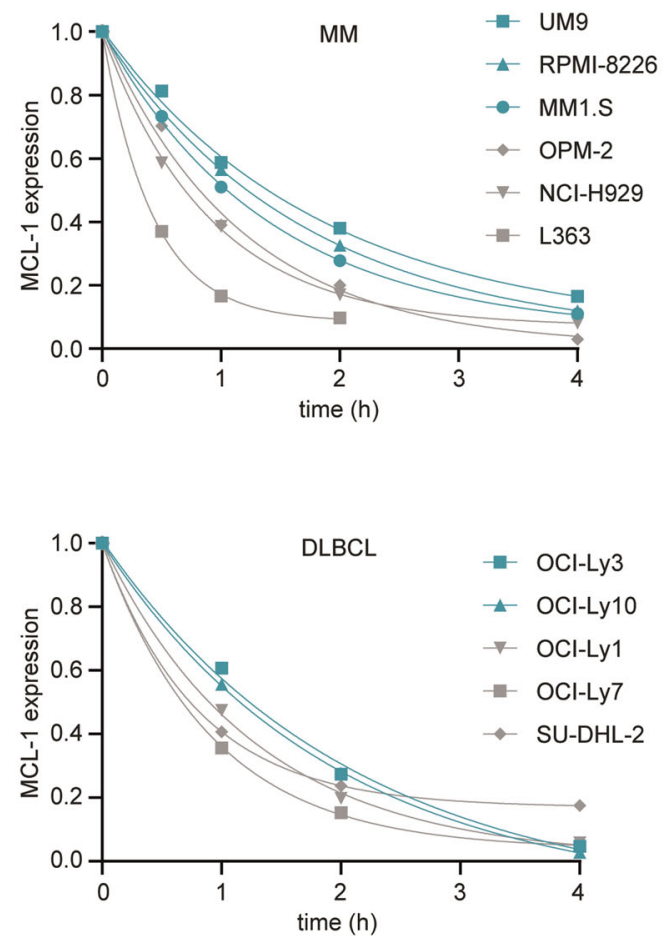

C

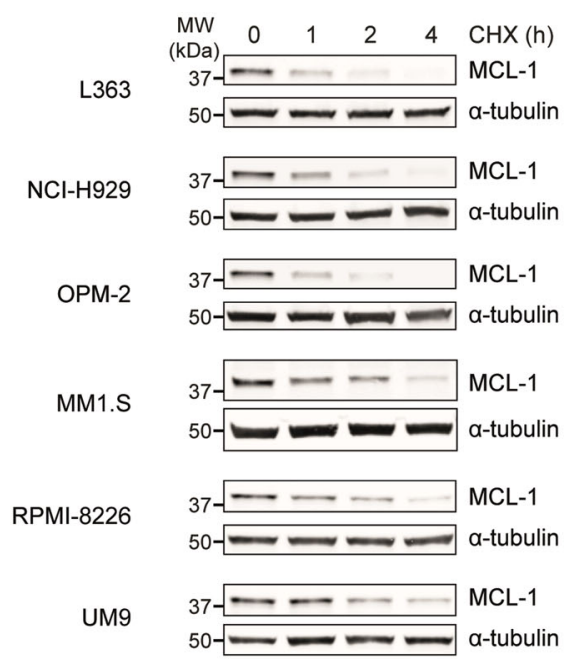

DLBCL
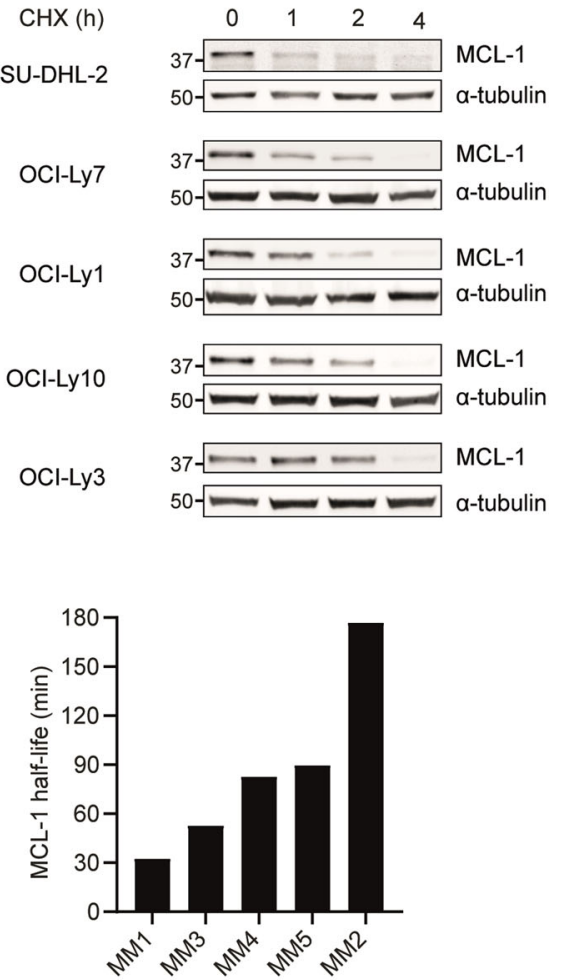

Fig. 1 MCL-1 is stabilized in post-GC B cell malignancies. A-C MCL-1 protein half-life as determined by treatment of MM and DLBCL cell lines with cycloheximide (CHX). Presented data are averages of 3-6 independent replicates per cell line ( $n=3$ for RPMI-8226, L363, OCI-Ly10, and OCI-Ly7; $n=4$ for OPM-2, OCI-Ly3, OCI-Ly1, and SU-DHL-2; $n=5$ for MM1.S, UM9; $n=6$ for NCI-H929). A Summarized data (mean + SEM) of MCL-1 half-life in $6 \mathrm{MM}$ and 5 DLBCL cell lines. ${ }^{*}$ indicates half-life significantly longer than $40 \mathrm{~min}$, based on the $95 \%$ confidence interval. B Representative

immunoblot images showing MCL-1 levels after CHX treatment for the indicated time periods. C Non-linear regression analysis (one-phase decay) of MCL-1 degradation over time after CHX treatment. D MCL-1 half-life in primary (CD138-purified) cells from 5 MM bone marrow aspirates, analyzed by non-linear regression analysis. 


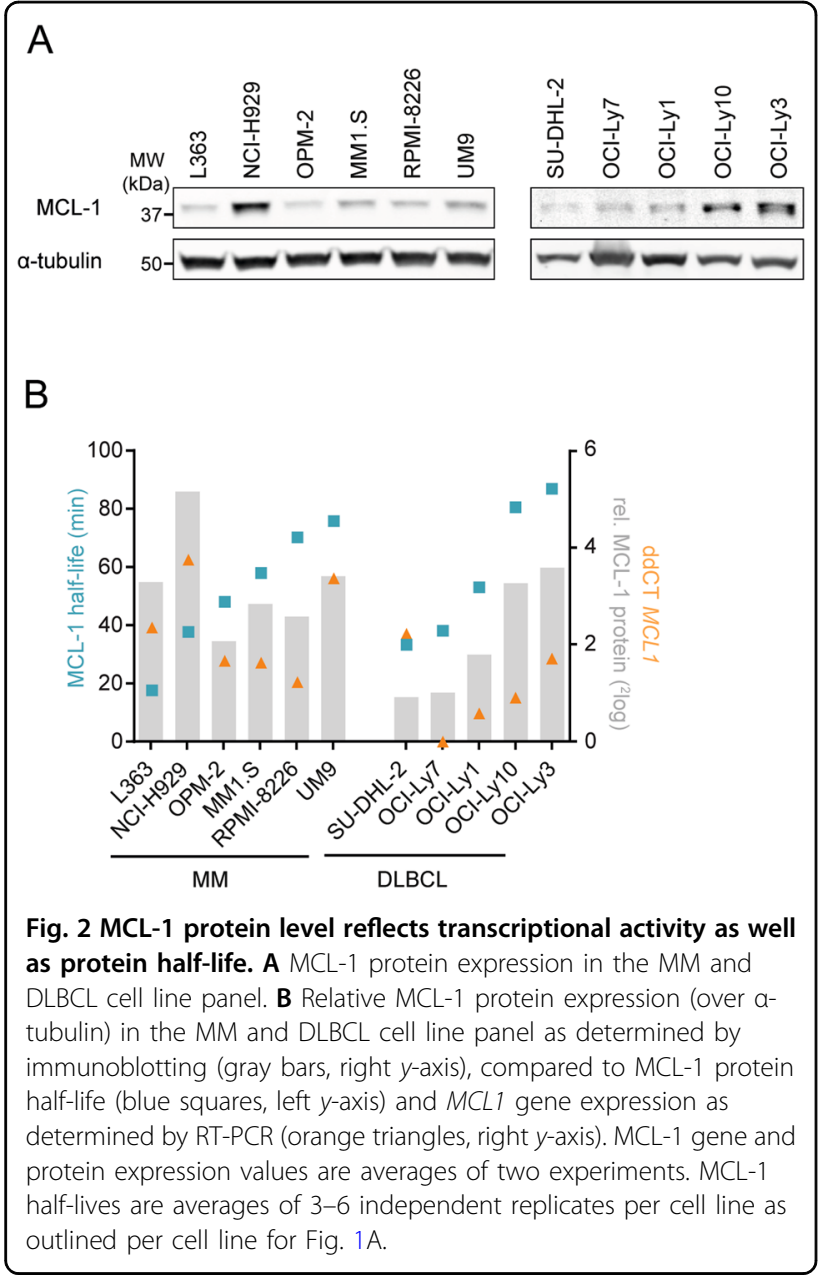

promote proteasomal degradation of MCL-1. Since the function of reported MCL-1-modifying proteins is highly cell type-specific, and no specific phosphatases have yet been linked to MCL-1 stabilization in MM, an unbiased approach was taken to identify phosphatases that dephosphorylate MCL-1 in MM. To this end, an siRNA screen targeting 188 phosphatases and phosphatase subunits was performed in MM cell line MM1.S (Fig. 3A). The screen was performed in three independent experiments, using altered MCL-1 protein expression as readout.

The phosphatase screen contained siRNAs against 188 phosphatases, 117 (62\%) of which were protein phosphatases (Fig. S1A). The top 10 hits identified in the screen (Fig. 3A) contained 7 protein phosphatases, of which 5 had Ser/Thr and 2 had dual phosphatase specificity (Fig. S1B). MCL-1 contains 13 reported putative phosphorylation sites in the PEST regions of the protein (Fig. S1C) $)^{20,36}$. Since 12 of these sites are Ser or Thr residues, the phosphatase that dephosphorylates MCL-1 is most likely to be of Ser/Thr or dual specificity. Among the top 10 phosphatases whose knockdown diminished
MCL-1 protein levels were three subunits of phosphatase complex PP2A (PPP2R1A, PPP2R2C, and PPP2R1B), and a PP2A-interacting protein (IGBP1; ref. ${ }^{37}$ ). Therefore, PP2A was identified as potential MCL-1-stabilizing phosphatase. PP2A is a Ser/Thr phosphatase complex consisting of three subunits: a catalytic, scaffold, and a regulatory subunit (Fig. 3B). Substrate specificity and affinity of PP2A is determined by the specific regulatory subunit present in the complex ${ }^{38}$. In the siRNA screen, both scaffold subunits PPP2R1A and PPP2R1B were identified, as well as one regulatory subunit (PPP2R2C).

\section{PP2A prevents MCL-1 degradation in MM with long MCL-1 half-life, but not in DLBCL}

To explore PP2A as potential MCL-1-stabilizing phosphatase, this phosphatase complex was further studied using OA, a small-molecule PP2A inhibitor. Treatment of MM and DLBCL cell lines with OA resulted in a reduction of MCL-1 in MM cell lines where MCL-1 has a long half-life, but not in cell lines where MCL-1 has a short half-life (Fig. 4A). Moreover, the reduction in MCL-1 was observed in none of the tested DLBCL cell lines, regardless of MCL-1 half-life, suggesting that MCL-1 stabilization in DLBCL may result from the action of other phosphatases or another mechanism altogether.

Immunoblot analysis revealed that the PP2A catalytic (C) subunit PPP2CA and scaffold (A) subunits PPP2R1A and PPP2R1B are present in all tested MM and DLBCL cell lines (Fig. 4B). Although no functional antibody was available to measure expression of regulatory subunit PPP2R2C by immunoblot, we did observe mRNA expression in MM cell lines with stabilized MCL-1 and in primary MM samples (Fig. S2). In fact, analysis of published data sets revealed that PPP2R2C gene expression was significantly higher in primary $M M$ compared to healthy GC B cells and higher compared to other GC B cell-derived malignancies (Fig. S3)

Due to the absence of MCL-1 degradation upon OA treatment in DLBCL, the effect of OA on MCL-1 was further studied only in MM.

RT-PCR analysis of OA-treated MM cell lines indicates that $\mathrm{OA}$ does not decrease transcriptional levels of $M C L 1$ (Fig. S4), suggesting that PP2A regulates MCL-1 protein stabilization rather than transcriptional regulation. In MM cell lines with short MCL-1 half-life, which are expected to rely more on MCL1 transcription than on protein stabilization, no reduction in MCL-1 was seen following OA treatment (Fig. 4A, C). To further show that MCL-1 protein expression in these cell lines is determined by gene transcription we tested the impact of an inhibitor of MCL-1 transcription. Cyclin-dependent kinase (CDK) 7 inhibition by THZ1 was previously shown to inhibit transcription of $M C L 1$, among other genes $^{39}$. As expected, treatment with THZ1 strongly 


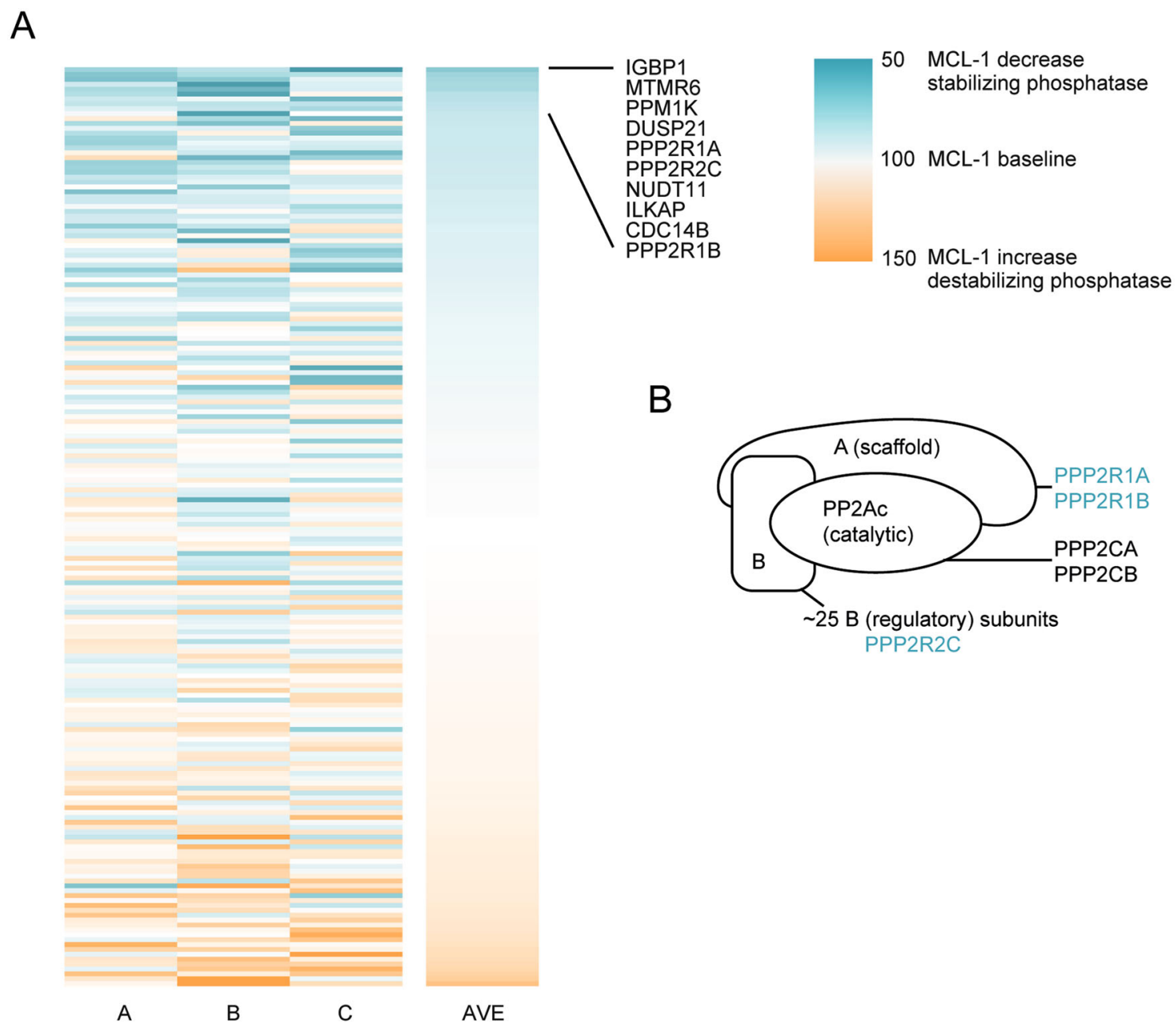

Fig. 3 Phosphatase siRNA screen identifies PP2A as MCL-1 stabilizing phosphatase. A Heatmap indicates changes in the MCL-1 level after phosphatase knockdown $(t=66 \mathrm{~h}$ ). The phosphatase siRNA screen was performed in three independent replicates (named A, B, or C), after which the 188 included siRNA pools were ranked based on average MCL-1 expression (AVE). The top 10 hits are named in the figure. B Schematic presentation of the three subunits of PP2A, with subunits that were identified in the phosphatase screen shown in blue.

reduced MCL-1 protein levels in L363, NCI-H929, and OPM-2 cells (Fig. 4C).

\section{PP2A inhibition leads to phosphorylation and destabilization of MCL-1}

MCL-1 degradation is thought to be preceded by phosphorylation, which may be reversed by PP2A. In concordance with this hypothesis, OA treatment increased MCL-1 phosphorylation at Ser159/Thr163 in MM cell lines with stabilized MCL-1, when the final step of MCL-1 degradation was prevented using a proteasome inhibitor. No enhanced MCL-1 phosphorylation was observed after treatment with TMC, a PP1 inhibitor (Fig. 5A).

Since these results indicate that PP2A dephosphorylates MCL-1, leading to increased MCL-1 protein levels, it is expected that OA treatment shortens MCL-1 half-life.
Indeed, CHX experiments reveal that OA reduces MCL-1 half-life, confirming that PP2A activity leads to increased MCL-1 stability in a subset of MM (Fig. 5B).

\section{JNK inhibition rescues MCL-1 destabilization after OA treatment}

Phosphorylation of MCL-1 on Ser155, Ser159, and Thr163 was previously reported to target MCL-1 for degradation. Our results indicating that PP2A inhibition leads to increased phosphorylation at Ser159/Thr163 (Fig. 5A) suggest that PP2A can counteract kinase activity at these particular phosphorylation sites. Kinases that have been shown to phosphorylate MCL-1 at Ser155, Ser159, and Thr163 are GSK-3 $\beta$, ERK-1, and JNK. In order to investigate whether one or more of these kinases is responsible for MCL-1 phosphorylation that is subsequently counteracted by $\mathrm{PP} 2 \mathrm{~A}$, we combined $\mathrm{OA}$ 


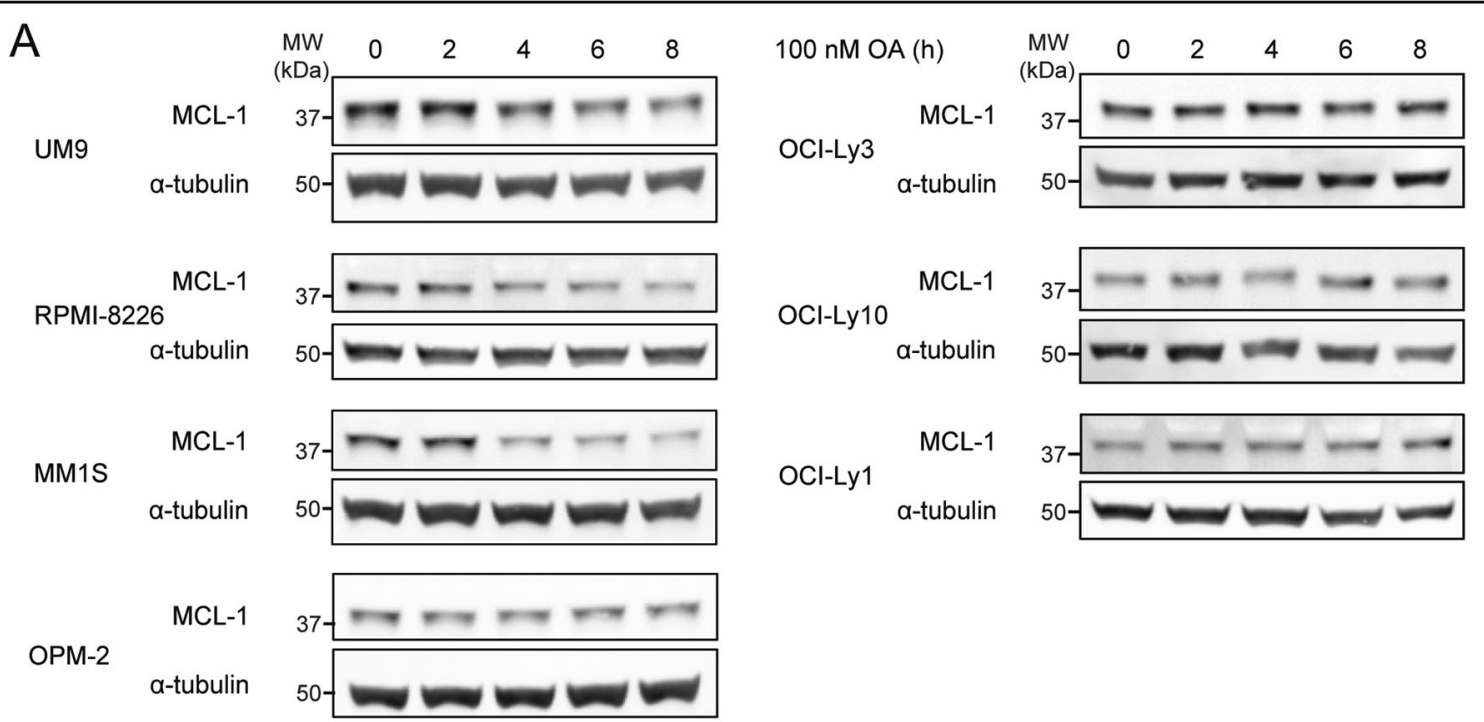

B

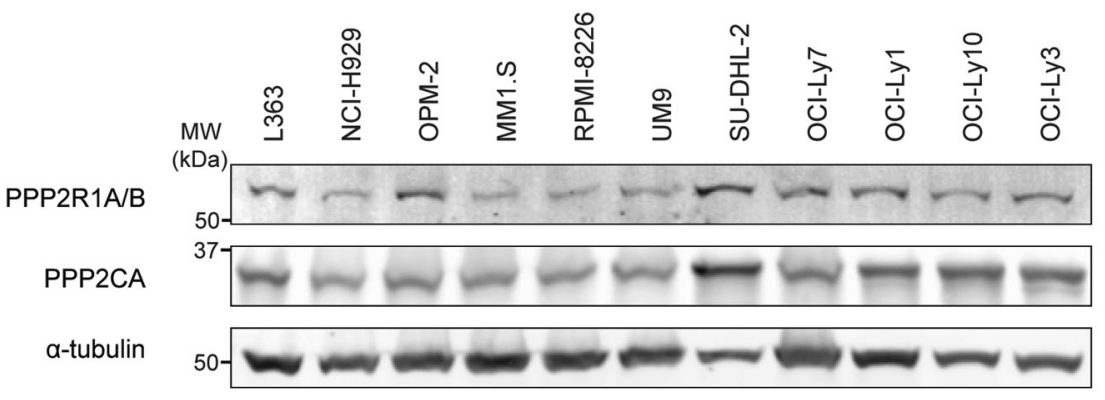

C

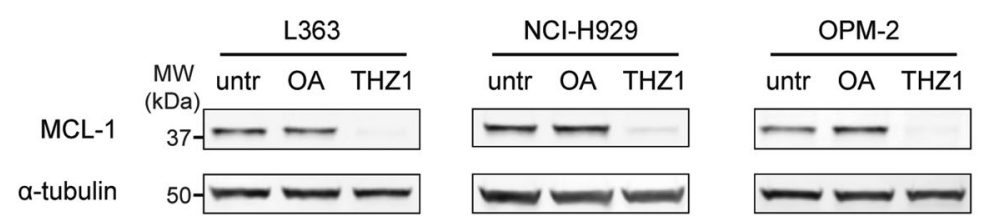

Fig. 4 PP2A prevents MCL-1 degradation in MM with long MCL-1 half-life, but not in DLBCL. A MCL-1 protein levels after treatment with $100 \mathrm{nM}$ PP2A inhibitor okadaic acid (OA) for the indicated time periods in MM cell lines (left) and DLBCL cell lines (right), in the presence of $10 \mu \mathrm{M}$ pan-caspase inhibitor Q-VD-OPh. B Expression of the PP2A catalytic subunit PPP2CA and scaffold subunits PPP2R1A/B in the MM and DLBCL cell line panel. C MCL-1 protein levels after $8 \mathrm{~h}$ treatment with $100 \mathrm{nM}$ of OA or $200 \mathrm{nM}$ of CDK7 inhibitor THZ1 in MM cell lines with short MCL-1 half-life.

treatment of MM1.S cells with specific kinase inhibition (Fig. 6A). GSK-3ß, ERK-1, and JNK were inhibited using CHIR99021, trametinib, or SP600125, respectively. Of these inhibitors, only JNK inhibitor SP600125 was able to rescue MCL-1 stability during OA treatment (Fig. 6B). Hence, PP2A stabilizes MCL-1 by counteracting phosphorylation by JNK, but not by GSK-3 $\beta$ or ERK-1.

\section{Discussion}

MCL-1 protein expression in MM cells is regulated at multiple levels. Firstly, overexpression can occur at the transcriptional level, resulting from signals from the bone marrow microenvironment or from amplification of 1q21, the locus that contains the MCL1 gene ${ }^{2,40} .1 \mathrm{q} 21$ amplification occurs in $\sim 30 \%$ of newly diagnosed and $\sim 70 \%$ of relapsed MM patients, and is associated with increased MCL1 expression as well as enhanced MCL-1 inhibitor sensitivity ${ }^{41,42}$. Secondly, alternative splicing and mRNAregulation can influence the amount of MCL-1 protein translation $^{43,44}$. Thirdly, MCL-1 is subject to heavy posttranslational regulation, which determines its stability and function $^{20}$.

Some phosphorylation sites of MCL-1 have been experimentally studied in cell types other than MM. In general, phosphorylation of MCL-1 at Thr163 is considered instrumental for regulation of protein stability and 
A

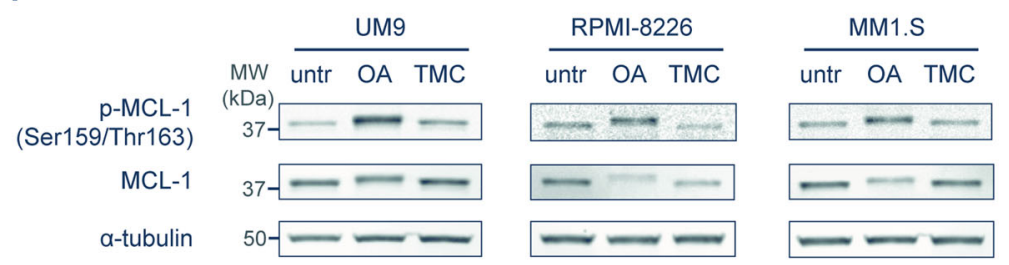

B
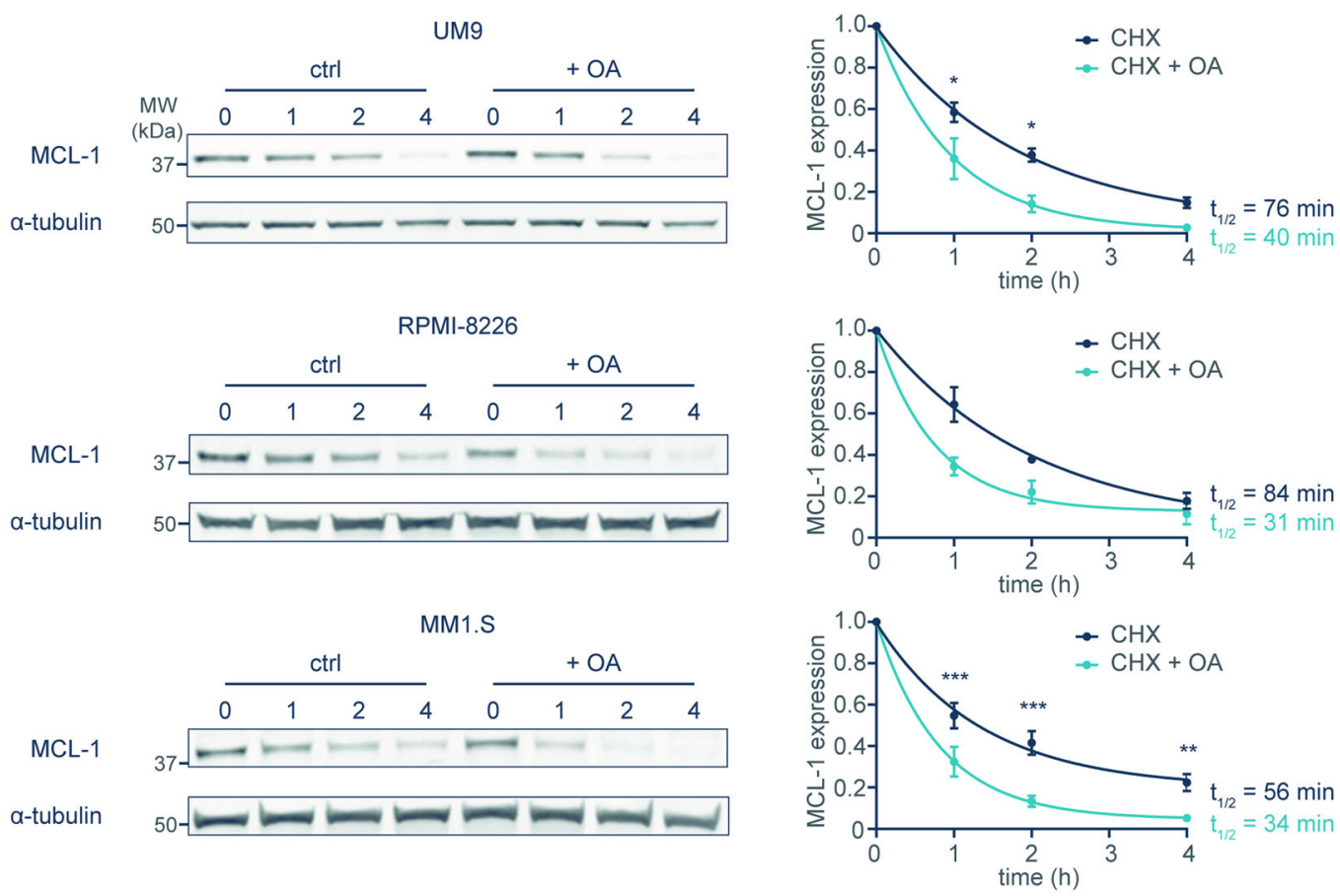

Fig. 5 PP2A inhibition leads to phosphorylation and destabilization of MCL-1. A Phosphorylation of MCL-1 (residues Ser159/Thr163) after treatment of MM cell lines with long MCL-1 half-life for $2 \mathrm{~h}$ with either $100 \mathrm{nM}$ OA or $1 \mu \mathrm{M} \mathrm{PP} 1$ inhibitor tautomycetin (TMC). All samples were treated in the presence of $20 \mathrm{nM}$ proteasome inhibitor bortezomib and $10 \mu \mathrm{M}$ Q-VD-OPh in order to prevent proteasomal degradation of phosphorylated MCL-1, while maintaining cell viability. B MCL-1 half-life after treatment with OA. Experiments and analyses were performed as in Fig. 1A-C. OAtreated samples received $2 \mathrm{~h}$ pretreatment with $\mathrm{OA}$, and $\mathrm{OA}$ remained present during $\mathrm{CHX}$ treatment. Graphs show averages of three independent replicates \pm SEM.

function, since this modification appears to be necessary for phosphorylation of other residues ${ }^{23}$. In conjunction with phosphorylation at Thr163, residues Thr92, Ser121, Ser155, and Ser159 can be phosphorylated. Phosphorylation of these sites has been associated with stabilization or destabilization of MCL-1 (refs. ${ }^{19,22-25,45}$ ). Based on our observation that PP2A dephosphorylates MCL-1 at Ser159/Thr163 (Fig. 5A), which was also shown by others in lymphoma cells ${ }^{46}$, we studied the kinases that can phosphorylate MCL-1 at these residues: GSK-3, ERK-1, and JNK. Of these kinases, only JNK inhibition was able to rescue MCL-1 stability upon OA treatment in MM (Fig. 6). Therefore, we conclude that JNK and PP2A together regulate MCL-1 protein stability in MM.
In contrast to the extensively studied MCL-1-modifying kinases, not much is known about the mechanisms of MCL-1 dephosphorylation. Classically, phosphatases are considered promiscuous and therefore less relevant to study in the context of specific cellular pathways than kinases. The genome encodes approximately threefold less phosphatases than kinases ${ }^{47,48}$, and phosphatases were thought to have a non-specific function in phosphoprotein homeostasis ${ }^{49}$. More recent findings, however, have shown that phosphatases play an active role in regulating cellular levels of tyrosine, threonine, and serine phosphorylation. Serine/threonine phosphatases such as PP2A act as holoenzymes containing a catalytic subunit, sometimes a scaffold subunit, and a regulatory subunit 
A
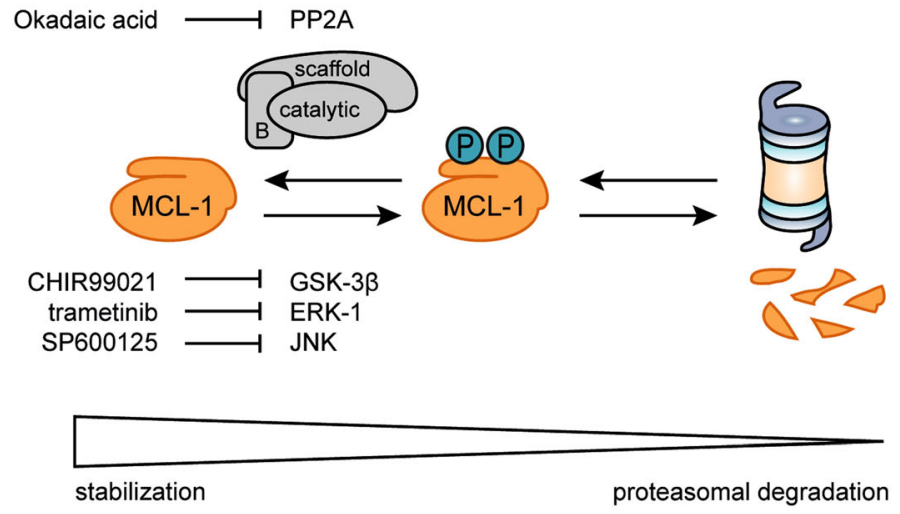

B
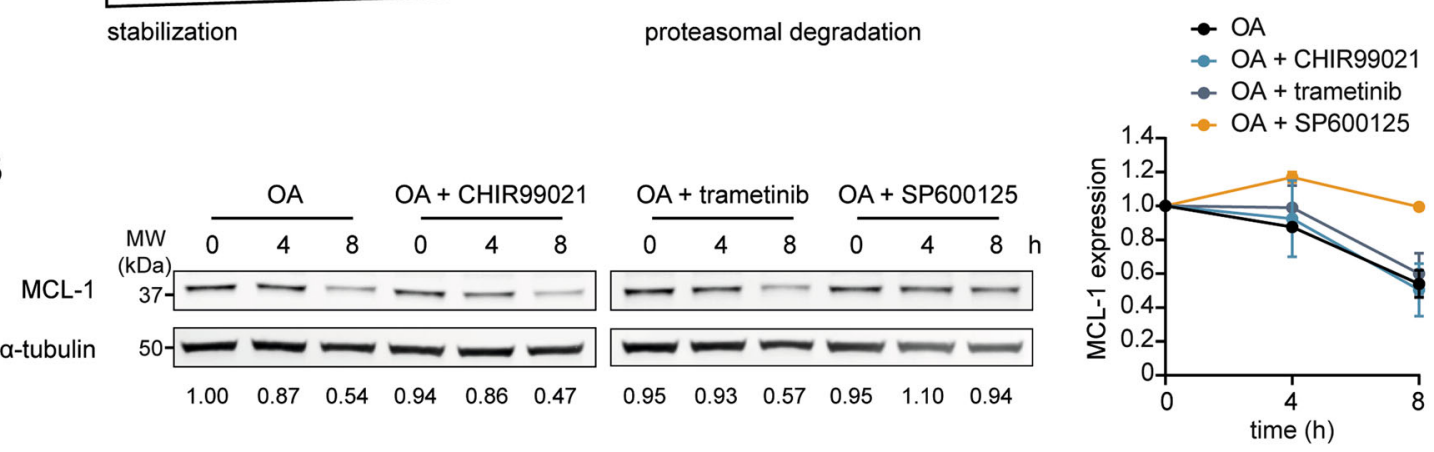

Fig. 6 PP2A reverses MCL-1 phosphorylation by JNK. A Model of MCL-1 phosphorylation leading to proteasomal degradation, which is counteracted in a subset of MM by PP2A phosphatase activity. Potential kinases phosphorylating and destabilizing MCL-1 are GSK-3 $\beta$, ERK-1, and JNK. The PP2A and kinase inhibitors used in this study are indicated. B MCL-1 expression in MM1.S after treatment for 0, 4, and $8 \mathrm{~h}$ with $100 \mathrm{nM}$ OA, either alone or combined with $10 \mu \mathrm{M}$ CHIR99021, $250 \mathrm{nM}$ trametinib, or 20 MM SP600125. Data represent two independent experiments. Quantified MCL-1 levels (normalized to a-tubulin level and relative to untreated) are shown below the bands and are average of two experiments. The graph shows averages of two independent replicates \pm SEM.

that recruits substrates to the complex ${ }^{38,50}$. As a result, PP2A can have many specific targets, depending on the regulation and availability of its regulatory subunits ${ }^{50}$.

We identified PP2A as the phosphatase that stabilizes MCL-1 in a subset of MM. In DLBCL, MCL-1 stabilization was observed in a subset of cell lines, but this did not result from PP2A activity. Immunoblotting indicated that the catalytic and scaffold subunits of PP2A are expressed in all tested MM and DLBCL cell lines (Fig. 4B). It is possible that the regulatory PP2A subunit responsible for MCL-1 targeting is lacking in DLBCL cells. The phosphatase siRNA screen identified one regulatory PP2A subunit, PPP2R2C. Unfortunately, we were unable to stain this subunit in immunoblot experiments, and we can therefore not validate whether PPP2R2C was a true hit from the screen responsible for MCL-1-specificity of PP2A. Regardless, MCL-1 stabilization in DLBCL likely results from a PP2A-independent mechanism, possibly by other phosphatases, by kinases, or at the ubiquitin level.

We observed increased MCL-1 stability in three out of six MM cell lines and in four out of five tested primary MM samples MCL-1 half-life exceeded the published $30-40 \mathrm{~min}^{18,19}$. In two cell lines with the shortest MCL-1 half-life, L363 and NCI-H929, the high MCL-1 protein levels likely result from high transcription of $M C L 1$. It is conceivable that different processes contribute to high MCL-1 protein levels in MM patients as well. Thus, besides 1q21-amplified MM, where MCL1 transcription is increased $^{41}$, there may also be MM patients with increased MCL-1 stability, as can be expected based on our findings with primary MM cells ex vivo.

In addition to PP2A subunits, IGBP1 (ImmunoglobulinBinding Protein 1, alternatively named Alpha 4), was identified in the screen. IGBP1 does not have intrinsic phosphatase activity but is a regulator of PP2A activity. IGBP1 binds catalytic PP2A subunits and protects them from proteasomal degradation, and removal of IGBP1 was shown to result in loss of PP2A expression, leading to apoptosis $^{37,51,52}$. The observation that IGBP1 knockdown in our screen consistently led to reduced MCL-1 levels therefore supports the hypothesis that PP2A stabilizes MCL-1.

Despite PP2A often being considered a tumor suppressor that is downregulated or mutated in cancer $^{53-56}$, PP2A activity is essential for cell survival and its inhibition is thus a promising anti-cancer strategy ${ }^{57}$. This paradoxical 
role of PP2A in cancer likely results from the plethora of different cellular functions in which PP2A is involved, depending on the exact composition of catalytic, scaffold, and regulatory domains. Although the development of specific phosphatase inhibitors lags behind kinase inhibitor development, increasing knowledge about phosphatase biology shows that it is possible to generate smallmolecule phosphatase inhibitors ${ }^{58}$. In a phase 1 clinical trial, the PP2A inhibitor LB100 was deemed safe to continue further development ${ }^{59}$. LB100 has strong chemoand radiosensitizing potential in cancer $^{57,60}$, indicating that PP2A inhibition could be a promising therapeutic strategy. LB100 was shown to overcome cellular senescence and promote mitotic catastrophe and apoptosis ${ }^{57}$. Because of the chemosensitizing potential of LB100, it would be highly relevant to address the interplay between PP2A inhibitors and established MM drugs. As an alternative to systemic inhibition of all PP2A, generation of a PP2A inhibitor that only inhibits the specific MCL-1-targeting regulatory subunit would be a promising future option.

In conclusion, we show that MCL-1 is stabilized in a subset of MM and DLBCL, and that PP2A is the phosphatase responsible for MCL-1 dephosphorylation and stabilization in MM. This finding increases the understanding of post-translational regulation of MCL-1, which may provide novel strategies to inhibit MCL-1 in MM cells.

\section{Acknowledgements}

The authors would like to thank the support facilities of the University Medical Center Utrecht (UMCU). This work was supported in part by a Bas Mulder Award from the Dutch Cancer Foundation (KWF)/Alpe d'HuZes Foundation (UU 2015-7663) (to V.P.) and grants from KWF/Alpe d'HuZes Foundation (11108 and 11270) (to V.P.).

\section{Author details}

${ }^{1}$ Center for Translational Immunology, University Medical Center Utrecht, Utrecht University, Utrecht, The Netherlands. ${ }^{2}$ Department of Experimental Immunology, Amsterdam University Medical Centers, University of Amsterdam, Amsterdam, The Netherlands. ${ }^{3}$ Cancer Center Amsterdam and LYMMCARE, Amsterdam, The Netherlands. ${ }^{4}$ Department of Hematology, Amsterdam. University Medical Centers, University of Amsterdam, Amsterdam, The Netherlands. ${ }^{5}$ Department of Hematology, University Medical Center Utrecht, Utrecht University, Utrecht, The Netherlands

\section{Author contributions}

A.S. and V.P. conceived the study and designed the experiments. E.E., M.J.K. and M.C.M. contributed to the design and interpretation of experiments. A.S. and L.M.M. performed the experiments. A.S., L.M.M., and V.P. contributed to the data collection and analysis. A.S. and V.P. wrote the manuscript. All authors read and approved the final manuscript.

\section{Conflict of interest}

The authors declare that they have no conflict of interest.

\section{Ethics statement}

Primary MM cell samples were derived from patients diagnosed at the Academic Medical Center, Amsterdam, The Netherlands. This study was conducted and approved by the AMC Medical Committee on Human
Experimentation. Informed consent was obtained in accordance with the Declaration of Helsinki.

\section{Publisher's note}

Springer Nature remains neutral with regard to jurisdictional claims in published maps and institutional affiliations.

Supplementary Information accompanies this paper at (https://doi.org/ 10.1038/s41419-020-03351-7).

Received: 18 May 2020 Revised: 10 December 2020 Accepted: 14 December 2020

Published online: 03 March 2021

\section{References}

1. Adams, J. M. \& Cory, S. The Bcl-2 apoptotic switch in cancer development and therapy. Oncogene 26, 1324-1337 (2007).

2. Slomp, A. \& Peperzak, V. Role and regulation of pro-survival BCL-2 proteins in multiple myeloma. Front. Oncol. 8, 533 (2018).

3. Zhang, B., Gojo, I. \& Fenton, R. G. Myeloid cell factor-1 is a critical survival factor for multiple myeloma. Blood 99, 1885-1893 (2002).

4. Derenne, S. et al. Antisense strategy shows that Mcl-1 rather than $\mathrm{BCl}-2$ or $\mathrm{BCl}-\mathrm{x}$ L is an essential survival protein of human myeloma cells. Blood 100, 194-199 (2002).

5. Tiedemann, R. E. et al. Identification of molecular vulnerabilities in human multiple myeloma cells by RNA interference lethality screening of the druggable genome. Cancer Res. 72, 757-768 (2012).

6. Wuillème-Toumi, S. et al. Mcl-1 is overexpressed in multiple myeloma and associated with relapse and shorter survival. Leukemia 19, 1248-1252 (2005).

7. Kotschy, A. et al. The MCL1 inhibitor S63845 is tolerable and effective in diverse cancer models. Nature 538, 477-482 (2016).

8. Caenepeel, S. et al. AMG 176, a selective MCL1 inhibitor is effective in hematological cancer models alone and in combination with established therapies. Cancer Discov. 8, 1582-1597 (2018).

9. Tron, A. E. et al. Discovery of Mcl-1-specific inhibitor AZD5991 and preclinical activity in multiple myeloma and acute myeloid leukemia. Nat. Commun. $\mathbf{9}$, 5341 (2018)

10. Peperzak, V. et al. Mcl-1 is essential for the survival of plasma cells. Nat Immunol. 14, 290-297 (2013).

11. Vikström, I. et al. Mcl-1 is essential for germinal center formation and B cell memory. Science 330, 1095-1100 (2010).

12. Vikström, I. et al. MCL-1 is required throughout B-cell development and its loss sensitizes specific B-cell subsets to inhibition of BCL-2 or BCL-XL. Cell Death Dis. 7. e2345 (2016).

13. Opferman, J. T. et al. Obligate role of anti-apoptotic MCL-1 in the survival of hematopoietic stem cells. Science 307, 1101-1105 (2005).

14. Thomas, R. L. et al. Loss of MCL-1 leads to impaired autophagy and rapid development of heart failure. Genes Dev. 27, 1365-1377 (2013).

15. Malone, C. D. et al. Mcl-1 regulates the survival of adult neural precursor cells, Mol. Cell. Neurosci. 49, 439-447 (2012).

16. Kozopas, K. M., Yang, T., Buchan, H. L., Zhou, P. \& Craig, R. W. MCL1, a gene expressed in programmed myeloid cell differentiation, has sequence similarity to BCL2. Proc. Natl Acad. Sci. USA 90, 3516-3520 (1993).

17. Yang, T., Kozopas, K. M. \& Craig, R. W. The intracellular distribution and pattern of expression of MCl-1 overlap with, but are not identical to, those of BCl-2. J. Cell Biol. 128, 1173-1184 (1995).

18. Nijhawan, D. et al. Elimination of Mcl-1 is required for the initiation of apoptosis following ultraviolet irradiation. Genes Dev. 17, 1475-1486 (2003).

19. Maurer, U., Charvet, C., Wagman, A. S., Dejardin, E. \& Green, D. R. Glycogen synthase kinase-3 regulates mitochondrial outer membrane permeabilization and apoptosis by destabilization of MCL-1. Mol. Cell 21, 749-760 (2006).

20. Thomas, L. W., Lam, C. \& Edwards, S. W. Mcl-1; the molecular regulation of protein function. FEBS Lett. 584, 2981-2989 (2010).

21. Mojsa, B., Lassot, I. \& Desagher, S. Mcl-1 ubiquitination: unique regulation of an essential survival protein. Cells 3, 418-437 (2014).

22. Ding, Q. et al. Down-regulation of myeloid cell leukemia-1 through inhibiting Erk/Pin 1 pathway by sorafenib facilitates chemosensitization in breast cancer. Cancer Res. 68, 6109-6118 (2008). 
23. Inoshita, S. et al. Phosphorylation and inactivation of myeloid cell leukemia 1 by JNK in response to oxidative stress. J. Biol. Chem. 277, 43730-43734 (2002).

24. Kodama, Y. et al. Antiapoptotic effect of c-Jun N-terminal Kinase-1 through Mcl-1 stabilisation in TNF-induced hepatocyte apoptosis. Gastroenterology 136 1423-1434 (2009).

25. Ding, Q. et al. Degradation of Mcl-1 by b-TrCP mediates glycogen synthase kinase 3-induced tumor suppression and chemosensitization. Mol. Cell. Biol. 27, 4006-4017 (2007).

26. Zhong, Q., Gao, W., Du, F. \& Wang, X. Mule/ARF-BP1, a BH3-only E3 ubiquitin ligase, catalyzes the polyubiquitination of $\mathrm{Mcl}-1$ and regulates apoptosis. Cell 121, 1085-1095 (2005).

27. Inuzuka, $\mathrm{H}$. et al. SCFFBW7 regulates cellular apoptosis by targeting MCL1 for ubiquitylation and destruction. Nature 471, 104-109 (2011).

28. Harley, M. E., Allan, L. A., Sanderson, H. S. \& Clarke, P. R. Phosphorylation of Mcl1 by $C D K-c y c l i n$ B1 initiates its Cdc20-dependent destruction during mitotic arrest. EMBO J. 29, 2407-2420 (2010).

29. Magiera, M. M. et al. Trim17-mediated ubiquitination and degradation of MCl-1 initiate apoptosis in neurons. Cell Death Differ. 20, 281-292 (2013).

30. Schwickart, M. et al. Deubiquitinase USP9X stabilizes MCL1 and promotes tumour cell survival. Nature 463, 103-107 (2010).

31. Wang, B. et al. Role of Ku70 in deubiquitination of Mcl-1 and suppression of apoptosis. Cell Death Differ. 21, 1160-1169 (2014).

32. Zhang, S. et al. Deubiquitinase USP13 dictates MCL1 stability and sensitivity to BH3 mimetic inhibitors. Nat. Commun. 9, 1-12 (2018).

33. Khalil, A. M., Cambier, J. C. \& Shlomchik, M. J. B cell receptor signal transduction in the GC is short-circuited by high phosphatase activity. Science $\mathbf{3 3 6}$ 1178-1182 (2012)

34. Peperzak, V., Slinger, E., Ter Burg, J. \& Eldering, E. Functional disparities among $\mathrm{BCL}-2$ members in tonsillar and leukemic B-cell subsets assessed by $\mathrm{BH} 3-$ mimetic profiling. Cell Death Differ. 24, 111-119 (2017).

35. Livak, K. J. \& Schmittgen, T. D. Analysis of relative gene expression data using realtime quantitative PCR and the 2- $\triangle \triangle C T$ method. Methods 25, 402-408 (2001)

36. Hornbeck, P. V. et al. PhosphoSitePlus, 2014: mutations, PTMs and recalibrations. Nucleic Acids Res. 43, D512-D520 (2015).

37. Kong, M., Ditsworth, D., Lindsten, T. \& Thompson, C. B. a4 is an essential regulator of PP2A phosphatase activity. Mol. Cell 36, 51-60 (2009).

38. Virshup, D. M. \& Shenolikar, S. From promiscuity to precision: protein phosphatases get a makeover. Mol. Cell 33, 537-545 (2009).

39. Huang, T. et al. CDK7 inhibitor THZ1 inhibits MCL1 synthesis and drives cholangiocarcinoma apoptosis in combination with BCL2/BCL-XL inhibitor ABT-263. Cell Death Dis. 10, 602 (2019).

40. Hanamura, I. et al. Frequent gain of chromosome band 1q21 in plasma-cell dyscrasias detected by fluorescence in situ hybridization: incidence increases from MGUS to relapsed myeloma and is related to prognosis and disease progression following tandem stem-cell transplantatio. Blood 108, 1724-1733 (2006).

41. Gupta, V. A. et al. Bone marrow microenvironment-derived signals induce Mcl-1 dependence in multiple myeloma. Blood 129, 1969-1979 (2017).
42. Slomp, A. et al. Multiple myeloma with amplification of chromosome $1 \mathrm{q}$ is highly sensitive to MCL-1 targeting. Blood Adv. 3, 4202-4214 (2019).

43. Cui, J. \& Placzek, W. J. Post-transcriptional regulation of anti-apoptotic BCL2 family members. Int. J. Mol. Sci. 19, 308 (2018)

44. Senichkin, V. V., Streletskaia, A. Y., Gorbunova, A. S., Zhivotovsky, B. \& Kopeina, G. S. Saga of Mcl-1: regulation from transcription to degradation. Cell Death Differ. 27, 405-419 (2020)

45. Morel, C., Carlson, S. M., White, F. M. \& Davis, R. J. Mcl-1 integrates the opposing actions of signaling pathways that mediate survival and apoptosis. Mol. Cell. Biol. 29, 3845-3852 (2009).

46. Nifoussi, S. K. et al. Inhibition of protein phosphatase 2A (PP2A) prevents Mcl-1 protein dephosphorylation at the Thr-163/Ser-159 phosphodegron, dramatically reducing expression in Mcl-1-amplified lymphoma cells. J. Biol. Chem. 289, 21950-21959 (2014).

47. Manning, G., Whyte, D. B., Martinez, R., Hunter, T. \& Sudarsanam, S. The protein kinase complement of the human genome. Science 298, 1912-1934 (2002).

48. Arena, S., Benvenuti, S. \& Bardelli, A. Genetic analysis of the kinome and phosphatome in cancer. Cell. Mol. Life Sci. 62, 2092-2099 (2005).

49. Sacco, F., Perfetto, L., Castagnoli, L. \& Cesareni, G. The human phosphatase interactome: an intricate family portrait. FEBS Lett. 586, 2732-2739 (2012).

50. Rowland, M. A., Harrison, B. \& Deeds, E. J. Phosphatase specificity and pathway insulation in signaling networks. Biophys. J. 108, 986-996 (2015).

51. Eleftheriadou, O. et al. Expression and regulation of type 2A protein phosphatases and alpha4 signalling in cardiac health and hypertrophy. Basic Res. Cardiol. 112, 37 (2017)

52. Kong, $\mathrm{M}$. et al. The PP2A-associated protein a4 is an essential inhibitor of apoptosis. Science 306, 695-698 (2004).

53. Seshacharyulu, P., Pandey, P., Datta, K. \& Batra, S. K. Phosphatase: PP2A structural importance, regulation and its aberrant expression in cancer. Cancer Lett. 335, 9-18 (2013).

54. O'Connor, C. M. et al. Inactivation of PP2A by a recurrent mutation drives resistance to MEK inhibitors. Oncogene 39, 703-717 (2020).

55. Sangodkar, J. et al. All roads lead to PP2A: exploiting the therapeutic potential of this phosphatase. FEBS J. 283, 1004-1024 (2016).

56. Eichhorn, P. J. A., Creyghton, M. P. \& Bernards, R. Protein phosphatase 2A regulatory subunits and cancer. Biochim. Biophys. Acta 1795, 1-15 (2009).

57. Hong, C. S. et al. LB100, a small molecule inhibitor of PP2A with potent chemo- and radio-sensitizing potential. Cancer Biol. Ther. 16, 821-833 (2015).

58. De Munter, S., Köhn, M. \& Bollen, M. Challenges and opportunities in the development of protein phosphatase-directed therapeutics. ACS Chem. Biol. 8 , 36-45 (2013).

59. Chung, V. et al. Safety, tolerability, and preliminary activity of LB-100, an inhibitor of protein phosphatase $2 \mathrm{~A}$, in patients with relapsed solid tumors: an open-label, dose escalation, first-in-human, phase I trial. Clin. Cancer Res. 23, 3277-3284 (2017)

60. Lai, D. et al. PP2A inhibition sensitizes cancer stem cells to ABL tyrosine kinase inhibitors in BCR-ABFL+ human leukemia. Sci. Transl. Med. 10, eaan8735 (2018). 